\title{
Systematic literature review of emergency department physicians' confidence to treat dental pain and the frequency with which they prescribe pain medications: Considerations for improved outcomes
} Sara Barna ${ }^{1}$, Jean O’Donnell $^{1}$, Marnie Oakley ${ }^{1}$

${ }^{1}$ University of Pittsburgh School of Dental Medicine, Pittsburgh, PA, USA

\section{Abstract}

Opioid analgesics, when taken as prescribed, are effective therapeutic options that provide pain relief for moderate to severe pain. The use of opioids in the treatment of pain has been increasing in the U.S. at an alarming rate, possibly contributing to the simultaneous rise in opioid abuse. Emergency departments play a major role in managing patients who present in pain, with approximately $10 \%$ of all opioid analgesic prescriptions written in hospital emergency departments. It is estimated that dental pain patients represent between $0.3-4 \%$ of the overall patient emergency department workload. Yet, the literature suggests that many of these physicians may not have sufficient training in handling dentofacial emergencies. The goal of this study was to systematically review the available literature on the topics of: 1) emergency department physicians' training related to treating dental pain patients, and 2) the frequency in which they prescribe opioid medications to these patients. Methods: $\mathrm{A}$ systematic literature review was conducted among publications from 1985-2014 in the databases PubMed, Ovid, and Science Citation Index. The following search terms were used in this systematic literature review in order to identify the available literature of interest: "opioid and dental and emergency departments," "dental pain and drug abuse," "ER physicians and dental pain," "ER physicians and drug abuse," and "dental pain and emergency departments." Publications in any language or country were considered, as well as editorials and commentaries. Findings: A total of 769 publications were identified. Seventeen publications met the criteria for inclusion. Eight studies commented on the emergency department physicians' perceived "lack of training" in handling dentofacial emergencies and found that the majority of this group did not feel comfortable in managing dental patients. Nine studies assessed the frequency in which physicians prescribe pain medications to dental patients. Within these 9 studies, 5 specifically reported that between $29.6 \%$ and $81 \%$ of dental patients treated, received an "opioid" or a "narcotic" upon discharge. The remaining 4 studies in this group instead used non-specific terms that included "prescription medications," "analgesics," "pain medicine," and "pharmacotherapy" to describe their findings and did not particularly report opioid prescribing trends. Of those publications rejected, 9 addressed the topic of dental pain patients presenting to non-dental providers, but did not include data that met the criteria related to emergency department prescribing frequency or physician training. Conclusions: This review of the literature suggests that emergency department physicians' training level in treating dentofacial pain is less than ideal. It also confirms that individuals presenting to emergency departments with dental pain are a subset of the population of patients who are prescribed opioids as an analgesic. Coupling these results with the increased use of opioids in this country, dentists are in a key position to collaborate with emergency department physicians to help positively affect change. To further justify this approach, research agendas must carefully monitor prescribing patterns for dentofacial pain in the emergency department that are specific to opioid use, carefully excluding other non-narcotic analgesics. Should comparable outcomes of data related to the same topic in other non-dental settings exist, additional areas in medicine that may benefit from this partnership may also be identified. Moving forward, this interprofessional team approach may include a presence in medical school and residency program curricula so that alternative treatment options for addressing dental pain patients can be presented that consider the increased prescribing trends of opioids.

Citation: Barna S, et al. (2019) Systematic literature review of emergency department physicians' confidence to treat dental pain and the frequency with which they prescribe pain medications: Considerations for improved outcomes. Dentistry 3000. 1:a001 doi:10.5195/d3000.2019.85

Received: August 17, 2018

Accepted: September 18, 2018

Published: June 26, 2019

Copyright: (C2019 Barna S, et al. This is an open access article licensed under a Creative Commons Attribution Work 4.0 United States License. Email: barnas1589@gmail.com

\section{Introduction}

Opioid analgesics, when taken as prescribed, are effective therapeutic options that provide adequate pain relief for moderate to severe pain. Prescription opioids are essential pain management tools; however, balancing their adverse effects and abuse potential with appropriate pain relief can be challenging (1). Recently, the increase in the prescribing of opioids for chronic non-malignant pain has increased accessibility, and most likely has contributed to the growing trend of nonmedical use as well as nonfatal and fatal overdoses (2). According to the
Center for Disease Control and Prevention (CDC) latest Policy Report, it is estimated that nearly 3 out of 4 prescription drug overdoses are caused by opioid pain relievers (3). In 2010, approximately 7.0 million people were current users of psychotherapeutic (pain relievers, tranquilizers, stimulants, 
sedatives) drugs taken nonmedically--nearly 3 percent of the U.S. population. (4). More than three out of four people who misuse prescription pain relievers use drugs prescribed to someone else (3). These trends continue to generate great concern among providers and government officials alike.

It has been estimated that approximately $10 \%$ of opioid analgesic prescriptions for people 20-39 years of age are written in hospital emergency departments (5). These emergency departments play an important role in the health care system and act as the "provider of last resort" for millions of Americans who lack adequate access to health care professionals, including dentists (4). And, although short-term analgesics are critical for acute pain management, long-term use of these medications creates a potential for substance abuse and drug-seeking behavior (6).

Many patients lack access to appropriate dental care due to barriers such as practice hours and lack of dental insurance (7). As a result, these patients often turn to medical providers when they experience dental pain (7). Several studies have shown that there has been a substantial increase in prescribing pain medications by non-dental providers $(6,8)$. In 2010 , there were approximately 129.8 million emergency department visits, and it is estimated that dentofacial emergencies represent between $0.3 \%$ and $4.0 \%$ of the overall patient emergency department workload $(9,7,5)$. Given these trends, emergency department providers should be equipped to diagnose, provide basic treatment, and ensure appropriate follow-up care for dental problems. Patients receiving limited and focused dental care in this setting could potentially decrease the need for prescribed opioid analgesics. However, the literature suggests that emergency department physicians do not feel confident in managing dentofacial emergencies and are interested in additional education $(9,10,7)$. These data suggest that emergency department providers may require more extensive training in alternative treatment procedures such as local anesthetic administration, nerve blocks or immobilization in order to respond to emergency department visits of dentofacial origin.

This paper systematically reviewed the available literature that relates to emergency department physicians' confidence in treating dental pain patients and the frequency with which they prescribe pain medication for these patients. Results are summarized and suggestions for improving outcomes are discussed.

\section{Methods}

The literature review by the author (SMB) included a search of PubMed, Ovid and Science
Citation Index databases using the keywords "dental pain and drug abuse," "ER physicians and dental pain," "ER physicians and drug abuse," and "dental pain and emergency departments" for studies published from 1985-2014. The following search terms were used because they provided the greatest number of publications pertaining to the topic.

Publications, editorials, and commentaries in any language or country regarding dental pain patients and the emergency department were considered. The editorials and commentaries that were included pertained information that was relevant to the topic, strengthening the (discussion) that emergency physicians may not feel confident handling dentofacial emergencies. In July of 2014, a total of 769 publications were identified. These publications were then reviewed and a total of seventeen publications were selected based on relevance of the title and abstract.

\section{Results}

Publications that commented on the emergency department physicians' clinical training related to treating dental pain patients:

Eight studies commented on emergency department physicians' perceived "lack of training" in handling dentofacial emergencies. The studies that had surveyed the physicians found that the majority of them did not feel comfortable in managing 
dental patients $(7,9)$. Of these 8 articles, however, only 2 articles reported statistical evidence where emergency department physicians did not feel confident in managing dentofacial emergencies. The other 6 articles stated that emergency department physicians lack training on treating dental pain patients, but did not survey the physicians in order to gather statistical evidence. The author of this systematic review (SMB) checked the references of these 8 articles in order to verify whether a referenced source had conducted a study and provided statistical evidence.

Trivedy et al. surveyed 103 emergency department physicians in various London hospitals as well others in the UK. The majority of participants $(76.5 \%)$ did not receive any clinical training in handling dental emergencies and indicated that they do not feel confident in managing certain dentofacial emergencies presenting to the emergency department. Skapetis et al. mentioned how "medical personnel lack both knowledge and training in dental emergencies." Furthermore, Skapetis et al. referenced a research article that surveyed 102 emergency medical personnel and found that $52 \%$ had no previous training in "examination of the mouth." Both of these studies were conducted outside of the United States. The other 6 publications did not survey physicians in order to determine if they were confident in treating dental pain patients in the emergency departments. Rather, they referenced other studies that surveyed physicians and/or referenced one another for the purpose of emphasizing the fact that emergency department physicians do not feel confident in handling dentofacial emergencies.

\section{Publications that assessed the}

frequency in which emergency department physicians prescribed pain medication for dental patients:

Nine studies assessed the frequency in which physicians prescribe pain medications to dental patients. Within these 9 studies, 5 specifically reported that between $29.6 \%$ and $81 \%$ of dental patients treated received an "opioid" or a "narcotic" upon discharge. The remaining 4 studies in this group instead used nonspecific terms that included "prescription medications," "analgesics," "pain medicine," and "pharmacotherapy" to describe their findings and did not particularly report opioid prescribing trends.

Of those publications rejected in this review, 9 addressed the topic of dental pain patients presenting to non-dental providers, but did not include data that met the criteria related to emergency department prescribing frequency or physician training. These studies mentioned information such as: techniques on how to manage dental patients in the emergency department, the national drug abuse problem, how dentists can help address the issue, and some of the more common dental emergencies (Figure 1).

Notably, according to the studies in Figure 1, emergency department physicians prescribe analgesics and/or opioids at variable frequencies. This could be contributed to factors such as the location of the study, the time frame, data collection methods, or the type of intervention that was conducted at each individual site. For example, Lewis et al looked at a 4-year period from 1997 to 2000 and found that approximately $72.4 \%$ of dental pain patients in the United States were prescribed an analgesic at the emergency department. These authors used data from the Center of Disease Control and Prevention. However, Okunseri et al looked at the time frame between 1997-2000 and 2003-2007 and found that dental pain patients in the emergency department were prescribed opioid analgesics only $43 \%$ of the time. These authors used data from the National Hospital Ambulatory Medical Care Survey.

\section{Limitations}

There were limitations to this review that should be noted. The authors of the reviewed 
publications varied in their description of "pain medication," therefore, the studies were not consistent in vocabulary used. The different terminology used without specifically stating the type of medication prescribed could result in an incorrect estimate of the frequency of prescribing pain medication (to include opioids) to dental pain patients in the emergency department. Additional studies with consistent definitions of pain medications are needed to fully understand the scope of the problem. There were few studies that met the criteria to be included in the systematic review, suggesting that more research is this area is warranted. Also, the time frame of the studies varied, which could account for the difference in the prescribing frequencies.

\section{Discussion}

Prescription drug abuse is a national epidemic and dentists as well as physicians are in key positions to help address this pertinent health care issue. The outcome of this systematic review reveals that there is an increasing trend in emergency department visits for dental issues, and that these patients are frequently treated with prescriptions for antibiotics and/or pain medication $(15,25)$. The underlying dental issue may never be addressed in any setting due to issues such as cost, access, insurance coverage, or dental treatment limitations. The following are suggestions targeted to improve oral health treatment of patients who present with dental pain to the emergency department. These suggestions will also help address the national drug abuse epidemic, while still achieving adequate pain relief for dental patients presenting to emergency departments.

One suggestion is to improve the oral health clinical training in medical school and residency programs to include other treatment options, such as administering local anesthetics through infiltration and/or nerve block techniques. The results from a national United States survey in 2009 found that most medical schools only offered 1-2 hours (28.4\%), or $3-4$ hours $(30.7 \%)$ of oral health curriculum during a student's four years of training. Furthermore, the same study notes that 1 in 10 schools offered no oral health curriculum (10.2\%) (30). Currently, there are no studies that have evaluated the application of hands-on clinical training to physicians in administering local anesthetics to dental pain patients in the emergency department. Administering local anesthetics coupled with prescribing nonopioid medications to dental pain patients in the emergency department can provide adequate relief until the patient can be evaluated by a dentist in a non- emergent setting at a later time. This technique may result in a decrease in prescribing opioids in the emergency department, yet still achieve effective pain relief for these dental pain patients (2).

Another suggestion is to implement prescribing guidelines into the emergency departments to assist emergency department physicians in treating dental pain patients. Some studies found that implementing prescribing guidelines drastically reduced the amount of opioids prescribed for patients presenting to the emergency department in pain (1, 13). Ma et al found that access to guidelines reduced narcotic prescriptions by $20.1 \%$. Gugelmann et al implemented a multifaceted educational program into 2 urban emergency departments, and the relative opioid discharge orders decreased by $39.6 \%$ and $56.2 \%$ for the primary and affiliated hospital, respectively. These guidelines highlight recent literature that supports using high doses of nonsteroidal anti-inflammatory drugs (NSAIDS) as an equally effective modality of care for patients with dental pain (2).

In summary, the literature suggests that the rate of prescribing pain medications to dental pain patients in the emergency department has been increasing $(8,6)$. A Center for Disease Control and Prevention 
(CDC) report from 2010 found that analgesics were the most mentioned medication class (i.e. medication given in the emergency department or prescribed at discharge) in the United States emergency departments at approximately $34.9 \%$. This number included narcotic and non-narcotic analgesics as well as NSAIDS (National Hospital Ambulatory Medical Care Survey: 2010 Emergency Department Summary Tables). Providing additional education to emergency department physicians may help bridge the gap in care for patients who present with dental pain. Through additional education, heightened awareness and/or guidelines for prescribing, physicians may be better equipped to address underlying dental problems of patients who present to emergency departments with dental pain.

\section{Acknowledgements}

S.B. was supported by the University of Pittsburgh School of Dental Medicine Dean's Summer Research program.

\section{References}

1. Multidisciplinary intervention decreases the use of opioid medication discharge packs from 2 urban EDs. Gugelmann $\mathrm{H}$, Shofer FS, Meisel ZF, Perrone J.Am J Emerg Med. 2013 Sep;31(9):1343-8. doi: 10.1016/j.ajem.2013.06.002
2. Prevention of prescription opioid abuse: the role of the dentist.

3. Denisco RC, Kenna GA, O'Neil MG, Kulich RJ, Moore PA, Kane WT, Mehta NR, Hersh EV, Katz NP. J Am Dent Assoc. 2011 Jul;142(7):800-10.

4. Policy Impact: Prescription Painkiller Overdoses. Centers for Disease Control and Prevention. Policy impact: prescription painkiller overdoses.

http://www.cdc.gov/homeandr ecreationalsafety/rxbrief/ Accessed August 4, 2014.

5. National Institute of Drug Abuse. Topics in brief: prescription drug abuse. http://www.drugabuse.gov/pu blications/topics-inbrief/prescription-drug-abuse. Accessed August 4, 2014.

6. Centers for Disease Control and Prevention. Addressing prescription drug abuse in the United States. 2013;1-36. http://www.cdc.gov/Homeand RecreationalSafety/pdf/HHS_Pr escription_Drug_Abuse_Report_ 09.2013.pdf. Accessed August 4, 2014.

7. Medications prescribed in emergency departments for nontraumatic dental condition visits in the United States. Okunseri C, Okunseri E, Thorpe JM, Xiang Q, Szabo A. Med Care. 2012 Jun;50(6):508-12. doi: 10.1097/MLR.0b013e318245a5 75.

8. Managing dental emergencies: a descriptive study of the effects of a multimodal educational intervention for primary care providers at six months. Skapetis T, Gerzina T, Hu W. BMC Med Educ. 2012 Oct 30;12:103. doi: $\quad 10.1186 / 1472-6920-12-$ 103.

9. Prescription of opioid and nonopioid analgesics for dental care in emergency departments: Findings from the National Hospital Ambulatory Medical Care Survey. Okunseri C, Okunseri E, Xiang Q, Thorpe JM, Szabo A. J Public Health Dent. 2014 Fall;74(4):283-92. doi: 10.1111/jphd.12055.

10. The attitudes and awareness of emergency department (ED) physicians towards the management of common dentofacial emergencies. Trivedy C, Kodate N, Ross A, AlRawi H, Jaiganesh T, Harris T, Anderson JE. Dent Traumatol. 2012 Apr;28(2):121-6. doi: 10.1111/j.16009657.2011.01050.x.

11. Expanding the physician's role in addressing the oral health of adults. Cohen LA. Am J Public Health. 2013 Mar;103(3):40812. 10.2105/AJPH.2012.300990

12. [11] Reducing the burden of dental patients on the busy hospital emergency department. McCormick AP, Abubaker AO, Laskin DM, Gonzales MS, Garland S. J Oral Maxillofac Surg. 2013 Mar;71(3):475-8. doi: 10.1016/j.joms.2012.08.023

13. Dental complaints in emergency departments: a national perspective. Lewis $\mathrm{C}$, Lynch $\mathrm{H}$, Johnston B. Ann Emerg Med. 2003 Jul;42(1):93-9.

14. Effect of education and guidelines for treatment of uncomplicated dental pain on patient and provider behavior. Ma M, Lindsell CJ, Jauch EC, Pancioli AM. Ann Emerg Med. 2004 Oct;44(4):323-9. 
15. Emergency department visits for dental care of nontraumatic origin. Quiñonez C, Gibson D, Jokovic A, Locker D. Community Dent Oral Epidemiol. 2009 Aug;37(4):366-71. doi: 10.1111/j.16000528.2009.00476.x.

16. Visiting the emergency department for dental problems: trends in utilization, 2001 to 2008. Lee HH, Lewis CW, Saltzman B, Starks H. Am J Public Health. 2012 Nov;102(11):e7783. doi: 10.2105/AJPH.2012.300965. Emergency dental anesthesia blocks. Daymude ML, Hilliard MW. Adv Emerg Nurs J. 2007;29(2):172-179.

17. Emergency department bane-dental pain used to obtain narcotics. Hupp JR. J Oral Maxillofac Surg. 2013 Dec;71(12):2009-10. doi: 10.1016/j.joms.2013.09.028

18. Doctor, my tooth hurts: the costs of incomplete dental care in the emergency room. Davis EE, Deinard AS, Maïga EW. J Public Health Dent. 2010 Summer;70(3):205-10. doi: $10.1111 /$ j.1752-

7325.2010.00166.x.

19. The root of the problem. Mckenna M. Ann Emerg Med. 2010;55(6):17-19.

20. Dental emergencies presenting to a general hospital emergency department in Hobart, Australia. Verma S, Chambers I. Aust Dent J. 2014 Sep;59(3):329-33. doi: 10.1111/adj.12202.

21. Managing dental pain in the emergency department: dental disparities with practice implications. Dowling Evans D, Gisness C. Adv Emerg Nurs J. 2013 Apr-Jun;35(2):95-102. doi:
10.1097/TME.0b013e31828f70

$1 \mathrm{e}$.

22. Cohen L, Bonito A, Eicheldinger $C$ et al. Comparison of patient visits to emergency departments, physician offices, and dental offices for dental problems and injuries. J Public Health Dent. 2011;71:13-22.

23. Visits to non-dentist health care providers for dental problems. Cohen LA, Manski RJ. Fam Med. 2006 Sep;38(8):556-64.

24. Pain and prescription monitoring programs in the emergency department. Todd KH. Ann Emerg Med. 2010 Jul;56(1):24-6. doi: 10.1016/j.annemergmed.2010. 02.025 .

25. Open wide! Dental settings are an untapped resource for substance misuse screening and brief intervention. McRee B. Addiction. 2012 Jul;107(7):1197-8. doi: 10.1111/j.13600443.2012.03777.x.

26. Dental visits to a North Carolina emergency department: a painful problem. Hocker MB, Villani JJ, Borawski JB, Evans CS, Nelson SM, Gerardo CJ, Limkakeng AT. N C Med J. 2012 Sep-Oct;73(5):346-51.

27. Dentistry in the emergency department. Gibson DE, Verono AA. J Emerg Med. 1987;5(1):3544.

28. Common dental emergencies. Evaluation and management for emergency physicians. Klokkevold P. Emerg Med Clin North Am. 1989 Feb;7(1):29-63.

29. Community-wide emergency department visits by patients suspected of drug-seeking behavior. Zechnich AD, Hedges
JR. Acad Emerg Med. 1996 Apr;3(4):312-7.

30. Teaching oral health in U.S. medical schools: results of a national survey. Ferullo A, Silk H, Savageau JA. Acad Med. 2011 Feb;86(2):226-30. doi: 10.1097/ACM.0b013e3182045a 51

31. A performance improvement prescribing guideline reduces opioid prescriptions for emergency department dental pain patients. Fox TR, Li J, Stevens S, Tippie T. Ann Emerg Med. 2013 Sep;62(3):237-40. doi:

10.1016/j.annemergmed.2012. 11.020 